\title{
A Single Species Model with Symmetric Bidirectional Impulsive Diffusion and Dispersal Delay
}

\author{
Haiyun Wan, Long Zhang*, Hongli Li \\ College of Mathematics and System Sciences, Xinjiang University, Urumqi, China \\ Email: ywansd@163.com, "longzhang_xj@sohu.com, lihongli3800087@163.com
}

Received August 3, 2012; revised September 3, 2012; accepted September 10, 2012

\begin{abstract}
In the natural ecosystem, impulsive diffusion provides a more natural description for population dynamics. In addition, dispersal processes often involve with time delay. In view of these facts, a single species model with impulsive diffusion and dispersal delay is formulated. By the stroboscopic map of the discrete dynamical system and other analysis methods, the permanence of the system is investigated. Moreover, sufficient conditions on the existence and uniqueness of a positive periodic solution for the system are derived from the intermediate value theorem. We also demonstrate the global stability of the positive periodic solution by the theory of discrete dynamical system. Finally, numerical simulations and discussion are presented to validate our theoretical results.
\end{abstract}

Keywords: Dispersal Delay; Impulsive Diffusion; Permanence; Global Stability; Periodic Solution

\section{Introduction}

Species dispersal in patchy environment is one of the most prevalent phenomena of nature, and many empirical works and monographs on population dynamics in a spatial heterogeneous environment have been done. The persistence and extinction for ordinary differential equation and delayed differential equation models were investigated in [1-6]. Global stability of equilibrium and periodic solution for diffusing model were studied in [7-12]. Particularly, the predator-prey system with the prey dispersal also were studied in [13-15]. Regretfully, in all of above population dispersing systems, they always assumed that the dispersal occurs at every time. For example, in [16], Mahbuba and Chen proposed the following two patches single species diffusion system:

$$
\begin{aligned}
\frac{\mathrm{d} x_{i}(t)}{\mathrm{d} t}= & x_{i}(t)\left[b_{i}(t)-a_{i}(t) x_{i}(t)\right] \\
& +D_{i j}(t)\left[x_{j}(t)-x_{i}(t)\right], i, j=1,2,
\end{aligned}
$$

where $D_{i j}(t)$ presents the dispersal rate from patch $j$ to patch $i$ at time $t$. The form of the dispersal established in this model is continuous, that is, the dispersal is always happening at any time.

Actually, real dispersal behavior is very complicated and is always influenced by environmental change and human activities. It usually occurs stochastically or discontinuously [17], and it is often the case that species dispersal occurs at some transitory time slots when indi-

${ }^{*}$ Corresponding author. viduals move among patches to search for mates, food, refuge, etc.

Animal movements between regions or patches of habitat are rarely continuous in time. They may occur during short time slots within seasons or within the lifetimes of animals. This short-time scale dispersal is more appropriately assumed to be in the form of impulses in the modeling process, in order to be in much better agreement with the real ecological situation. For example, when winter comes, birds will migrate between patches in search for a better environment, whereas they do not diffuse in other season, and the excursion of foliage seeds occurs during a fixed period of time every year. Thus impulsive dispersal provides a more natural description. With the developments and applications of impulsive differential equations, theories of impulsive differential equations have been introduced into population dynamics, and many important studies have been performed [18-24].

In [19], Wang and Chen studied the following autonomous single-species model with impulsively bidirectional diffusion:

$$
\left\{\begin{array}{l}
\frac{\mathrm{d} N_{1}(t)}{\mathrm{d} t}=r_{1} N_{1}(t) \ln \frac{k_{1}}{N_{1}(t)}, \\
\frac{\mathrm{d} N_{2}(t)}{\mathrm{d} t}=r_{2} N_{2}(t) \ln \frac{k_{2}}{N_{2}(t)}, \quad t \neq n \tau, \\
\Delta N_{1}(t)=d_{1}\left[N_{2}(t)-N_{1}(t)\right], \\
\Delta N_{2}(t)=d_{2}\left[N_{1}(t)-N_{2}(t)\right], \quad t=n \tau,
\end{array}\right.
$$


where $N_{i}(t) \quad(i=1,2)$ presents the population density or size at time $t, r_{i}(i=1,2)$ is the intrinsic growth rate of the population $N_{i}$, and $d_{i}$ is the dispersal rate in the ith patch. $\Delta N_{i}=N_{i}\left(n \tau^{+}\right)-N_{i}\left(n \tau^{-}\right)$, where $N_{i}\left(n \tau^{+}\right)$ represents the density of population in the ith patch immediately after the $n$th diffusion pulse at time $t=n \tau$, while $N_{i}\left(n \tau^{-}\right)$represents the density of population in the $i$ th patch before the $n$th diffusion pulse at time $t=n \tau \quad$ ( $\tau$ the period of dispersal between any two pulse events is a positive constant, $n=1,2, \cdots)$. It is assumed here that the net exchange from the $j$ th patch to $i$ th patch is proportional to the difference $N_{j}-N_{i}$ of population densities. The dispersal behavior of populations between two patches occurs only at the impulsive instants $n \tau$. Obviously, in this model, species $N$ inhabits respectively two patches before the pulse appears, when the time at the pulse comes, species $N$ both in two patches disperse from one patch to the other. Sufficient criteria were obtained for the permanence, existence, uniqueness and global stability of positive periodic solutions by using discrete dynamical system theory.

It is well known that the time delay is quite common for a natural population. In order to reflect the dynamical behaviors of models that depend on the past history of system, it is often necessary to take the effect of time delay into account in forming a biologically meaningful mathematica model. Delay differential equations have attracted a significant interest in recent years due to their frequent appearance in a wide range of applications. They serve as mathematical models describing various phenomena in physics, biology, physiology, and engineering, see, e.g., $[25,26]$ and references therein. There has been an extensive theoretical works on delay differential equations in the past three decades. The research topics include global asymptotic stability of equilibria, existence of periodic solutions, complicated behavior and chaos, see, e.g., $[8,14,27,28]$.

Zhang and Teng in [14] introduced the following two species time-delayed predator-prey Lotka-Volterra type dispersal system with periodic coefficients (1.3):

where $t \in R_{+}=[0, \infty), x_{i}(i \in I=\{1,2, \cdots, n\})$ denote the population density of prey species in $i$ th patch and $y$ is the population density of predator species. In this paper, the authors took dispersal delay into account, however, they assumed that the dispersal is continuous. Sufficient conditions on the boundedness, permanence and existence of positive periodic solution for system (1.3) are established.

Recently, the application of impulsive delay differential equations to population dynamics is also an interesting topic since it is reasonable and correct in modelling the evolution of population, such as pest management [29].

However, in all of the impulsive dispersal models studied up to now, there are few papers considering the dispersal delay, which is really a pity. Actually, in the real world, the migration between patches is usually not immediate, that is, dispersal processes often involve with time delay. For example, elks move from higher to lower elevations to escape cold in winter, and ungulates migrate annually among grazing areas to following spatio-temporal changes in rainfall. In these cases, movement is unidirectional during each migration period and takes place over fairly short time periods. Obviously, this kind of dispersal delay between patches extensively exists in the real world. Therefore, it is a very basilic problem to research this kind of population dynamic systems.

Motivated by the calculation hereinbefore, in this paper, we consider the following impulsive dispersal system with dispersal delay:

$$
\begin{cases}\frac{\mathrm{d} N_{1}(t)}{\mathrm{d} t}=r_{1} N_{1}(t) \ln \frac{k_{1}}{N_{1}(t)}, & t \neq n \tau, \\ \frac{\mathrm{d} N_{2}(t)}{\mathrm{d} t}=r_{2} N_{2}(t) \ln \frac{k_{2}}{N_{2}(t)}, & \\ \Delta N_{1}(t)=d_{1}\left[N_{2}(t-\tilde{\tau})-N_{1}(t)\right], & \\ \Delta N_{2}(t)=d_{2}\left[N_{1}(t-\tilde{\tau})-N_{2}(t)\right], & t=n \tau,\end{cases}
$$

where we suppose that the system is composed of two patches connected by diffusion. The pulse diffusion occurs every $\tau$ period, the system evolves from its initial state without being further affected by diffusion until the next pulse appears; $\tilde{\tau}$ stands for the time delay, that is, a period of species $N$ disperse between patch $i$ and $j$. $0<\tilde{\tau}<\tau, r_{i}, k_{i}$ and $d_{i}(i=1,2)$ are positive constants.

The organization of this paper is as follows. In section 2 , as preliminaries, the definition of permanence and some useful lemmas are introduced. From discrete dynamic system theory, we establish the stroboscopic map of system (1.4), by which we can obtain the dynamical

$$
\left\{\begin{array}{l}
\frac{\mathrm{d} x_{1}(t)}{\mathrm{d} t}=x_{1}(t)\left[a_{1}(t)-b_{1}(t) x_{1}(t)-c(t) \int_{-\infty}^{0} k_{12}(s) y(t+s) \mathrm{d} s\right]+\sum_{j=1}^{n}\left[\alpha_{1 j}(t) d_{1 j}(t) x_{j}\left(t-\tau_{1 j}(t)\right)-d_{j 1}(t) x_{1}(t)\right], \\
\frac{\mathrm{d} x_{i}(t)}{\mathrm{d} t}=x_{i}(t)\left[a_{i}(t)-b_{i}(t) x_{i}(t)\right]+\sum_{j=1}^{n}\left[\alpha_{i j}(t) d_{i j}(t) x_{j}\left(t-\tau_{i j}(t)\right)-d_{j i}(t) x_{i}(t)\right], \\
\frac{\mathrm{d} y(t)}{\mathrm{d} t}=y(t)\left[-e(t)+f(t) \int_{-\infty}^{0} k_{21}(s) x_{1}(t+s) \mathrm{d} s\right], i=2,3, \cdots, n,
\end{array}\right.
$$


behaviors of it. The theorem on the permanence for system (1.4) is stated and proved in Section 3. In section 4, the existence and uniqueness of positive periodic solution for the system are obtained by the intermediate value theorem. In Section 5, the global stability of the positive periodic solution for system is established by the discrete dynamic system theory in [30]. Finally, we give a brief discussion and our theoretical results are conformed by numerical simulations.

\section{Preliminaries}

For any fixed $t \in R_{+}=[0, \infty)$, let $\phi(s):[t-\tilde{\tau}, t] \rightarrow R^{2}$ be a piecewise continuous function such that $\phi(s)$ is continuous in $s \neq n \tau, \phi\left(n \tau^{-}\right)=\lim _{s \rightarrow n \tau^{-}} \phi(s)=\phi(n \tau)$ and $\phi\left(n \tau^{+}\right)=\lim _{s \rightarrow n \tau^{+}} \phi(s)$ exist. For any fixed $t \in R_{+}$, let $\mathrm{PC}[t-\tilde{\tau}, t]$ denote the Banach space of all such piecewise continuous functions $\phi(\theta):[t-\tilde{\tau}, t] \rightarrow R^{2}$ with the norm $\|\phi\|=\sup _{t-\tilde{\tau} \leq \theta \leq t}|\phi(\theta)|$. Further, let $\mathrm{PC}_{+}[t-\tilde{\tau}, t]=\left\{\phi=\left(\phi_{1}, \phi_{2}\right) \in \mathrm{PC}[t-\tilde{\tau}, t]: \phi_{i}(\theta) \geq 0\right.$ for all $\theta \in[t-\tilde{\tau}, t]$ and $\phi_{i}(t)>0$ for $\left.i=1,2\right\}$.

Motivated by the biological background of system (1.4), in this paper we always assume that all solutions of system (1.4) satisfy the following initial conditions:

$$
N_{i}(\theta)=\phi_{i}(\theta) \text { for all } \theta \in[-\tilde{\tau}, 0], i=1,2,
$$

where $\phi=\left(\phi_{1}, \phi_{2}\right) \in \mathrm{PC}_{+}[-\tilde{\tau}, 0]$. For any $\phi \in \mathrm{PC}_{+}[-\tilde{\tau}, 0]$, by the fundamental theory of impulsive functional differential equations [31,32], system (1.4) has a unique solution $N(t, \phi)=\left(N_{1}(t, \phi), N_{2}(t, \phi)\right)$ satisfying the initial conditions (2.1). It is easy to prove that the solution $N(t, \phi)$ of system (1.4) is positive, that is, $N_{i}(t, \phi)>0$ $(i=1,2)$ in its maximal interval of the existence.

Definition 2.1. System (1.4) is said to be permanent, if there are positive constants $m_{i}$ and $M_{i}$ such that

$$
m_{i} \leq \liminf _{t \rightarrow \infty} N_{i}(t) \leq \limsup _{t \rightarrow \infty} N_{i}(t) \leq M_{i}, i=1,2,
$$

for any positive solution $N(t)=\left(N_{1}(t), N_{2}(t)\right)$ of system (1.4).

Next, to study the permanence, existence and uniqueness of positively periodic solutions for system (1.4), we take $x=\frac{N_{1}}{k_{1}}, y=\frac{N_{2}}{k_{2}}, k=\frac{k_{2}}{k_{1}}$, which on substituting into (1.4) becomes:

$$
\begin{cases}\frac{\mathrm{d} x(t)}{\mathrm{d} t}=r_{1} x(t) \ln \frac{k_{1}}{x(t)}, & \\ \frac{\mathrm{d} y(t)}{\mathrm{d} t}=r_{2} y(t) \ln \frac{k_{2}}{y(t)}, & t \neq n \tau, \\ \Delta x(t)=d_{1}[k y(t-\tilde{\tau})-x(t)], & \\ \Delta y(t)=d_{2}\left[\frac{1}{k} x(t-\tilde{\tau})-y(t)\right], & t=n \tau, n=1,2, \cdots\end{cases}
$$

By integrating and solving the first two equations of system (2.2) between pulse, we have

$$
\left\{\begin{array}{l}
x(t)=x_{n}^{e^{-n(t-n \tau)},}, \\
y(t)=y_{n}^{e^{-r_{2}(t-n \tau)}},
\end{array} \quad n \tau<t \leq(n+1) \tau .\right.
$$

Similarly, considering the last two equations of system (2.2), we obtain the following stroboscopic map of system (2.2):

$$
\left\{\begin{array}{l}
x_{n+1}=\left(1-d_{1}\right) x_{n}^{b_{1}}+d_{1} k y_{n}^{b_{2} e^{r^{2} \tilde{\tau}}}, \\
y_{n+1}=\left(1-d_{2}\right) y_{n}^{b_{2}}+\frac{d_{2}}{k} x_{n}^{b_{1} e^{n \tilde{\tau}}},
\end{array}\right.
$$

where

$$
\begin{gathered}
x_{n}=x\left(n \tau^{+}\right), y_{n}=y\left(n \tau^{+}\right), \\
0<b_{1}=e^{-r_{1} \tau}<1,0<b_{2}=e^{-r_{2} \tau}<1 .
\end{gathered}
$$

Remark 2.1. System (2.4) is a difference system. It describes the densities of population in two patches at a pulse in terms of values at the previous pulse. We are, in other words, stroboscopically sampling at its pulsing period. The dynamical behavior of system (2.4), coupled with (2.3), determine the dynamical behavior of system (1.4). In the following sections, we will focus our attention on system (2.4), and investigate various aspects of its dynamical behavior.

Next, In order to establish the permanence of system (2.4), we introduce the following two lemmas.

Lemma 2.1 Without loss of generality we assume that $k \leq 1$. Let $a_{1}=\min \left\{b_{1}, b_{2}\right\}, \quad a_{2}=\max \left\{b_{1} e^{r_{1} \tilde{\tau}}, b_{2} e^{r_{2} \tilde{\tau}}\right\}$. For $\forall x_{0}>0, y_{0}>0$, if $0<d_{1}+d_{2}<1$, then we have:

Case (1). If $x_{0} \leq y_{0}, y_{0} \leq 1$, then $x_{n} \leq y_{0}^{a_{1}^{n}}, y_{n} \leq \frac{1}{k} y_{0}^{a_{1}^{n}}$, $n=1,2, \cdots$; if $x_{0} \leq y_{0}, y_{0}>1$, then $x_{n} \leq y_{0}^{a_{2}^{n}}, \quad y_{n} \leq \frac{1}{k} y_{0}^{a_{2}^{n}}$, $n=1,2, \cdots$.

Case (2). If $x_{0}>y_{0}, x_{0} \leq 1$, then $x_{n} \leq x_{0}^{a_{1}^{n}}, y_{n} \leq \frac{1}{k} x_{0}^{a_{1}^{n}}$, $n=1,2, \cdots$; if $x_{0}>y_{0}, x_{0}>1$, then $x_{n} \leq x_{0}^{a_{2}^{n}}, y_{n} \leq \frac{1}{k} x_{0}^{a_{2}^{n}}$, $n=1,2, \cdots$.

Proof. We first prove that the first part of Case (1) holds. If $x_{0} \leq y_{0}, y_{0} \leq 1$, then from (2.4), we have

$$
\begin{aligned}
x_{1} & =\left(1-d_{1}\right) x_{0}^{b_{1}}+d_{1} k y_{0}^{b_{2} e^{r^{2} \tau}} \\
& \leq\left(1-d_{1}\right) y_{0}^{b_{1}}+d_{1} k y_{0}^{b_{2} e^{r_{2}} \tilde{z}} \\
& \leq\left(1-d_{1}\right) y_{0}^{b_{1}}+d_{1} k y_{0}^{b_{2}} \\
& \leq\left(1-d_{1}\right) y_{0}^{a_{1}}+d_{1} y_{0}^{a_{1}} \\
& =y_{0}^{a_{1}}
\end{aligned}
$$


and

$$
\begin{aligned}
y_{1} & =\frac{d_{2}}{k} x_{0}^{b_{1} e^{e^{n} \tilde{\tau}}}+\left(1-d_{2}\right) y_{0}^{b_{2}} \\
& \leq \frac{d_{2}}{k} y_{0}^{b_{1} e^{r \tilde{\tau}}}+\left(1-d_{2}\right) y_{0}^{b_{2}} \\
& \leq \frac{d_{2}}{k} y_{0}^{b_{1}}+\frac{1}{k}\left(1-d_{2}\right) y_{0}^{b_{2}} \\
& \leq \frac{d_{2}}{k} y_{0}^{a_{1}}+\frac{1}{k}\left(1-d_{2}\right) y_{0}^{a_{1}} \\
& =\frac{1}{k} y_{0}^{a_{1}} .
\end{aligned}
$$

Hence, the first part of Case (1) is true for $n=1$. Next, we assume that the conclusion holds for $n=m>1$, that is,

$$
x_{m} \leq y_{0}^{a_{1}^{m}}, \quad y_{m} \leq \frac{1}{k} y_{0}^{a_{1}^{m}} .
$$

Then, for $n=m+1$, we get

$$
\begin{aligned}
& x_{m+1}=\left(1-d_{1}\right) x_{m}^{b_{1}}+d_{1} k y_{m}^{b_{2} e^{r_{2}} \tilde{\tau}} \\
& \leq\left(1-d_{1}\right) y_{0}^{b_{1} a_{1}^{m}}+d_{1} k\left(\frac{1}{k}\right)^{b_{2} e^{r_{2} \tilde{z}}} y_{0}^{a_{1}^{m} b_{2} e^{r_{2} \tilde{\tau}}} \\
& \leq\left(1-d_{1}\right) y_{0}^{a_{1}^{m+1}}+d_{1} k^{1-b_{2} e^{r 2^{2}}} y_{0}^{a_{1}^{m+1}} \\
& \leq\left(1-d_{1}\right) y_{0}^{a_{1}^{m+1}}+d_{1} y_{0}^{a_{1}^{m+1}} \\
& =y_{0}^{a_{1}^{m+1}} \text {, } \\
& y_{m+1}=\frac{d_{2}}{k} x_{m}^{b_{1} \eta^{\eta^{\tilde{\tau}}}}+\left(1-d_{2}\right) y_{m}^{b_{2}} \\
& \leq \frac{d_{2}}{k} y_{0}^{a_{1}^{m} b_{1} e^{n \tilde{\tau}}}+\left(1-d_{2}\right)\left(\frac{1}{k}\right)^{b_{2}} y_{0}^{b_{2} a_{1}^{m}} \\
& \leq \frac{d_{2}}{k} y_{0}^{a_{1}^{m+1}}+\left(1-d_{2}\right) \frac{1}{k} y_{0}^{a_{1}^{m+1}} \\
& =\frac{1}{k} y_{0}^{a_{1}^{m+1}} \text {. }
\end{aligned}
$$

From the above discussion, we can see that the first part of Case (1) is true. Similarly, we can prove that the second part of Case (1) and the Case (2) are also true, so we omit the proof. This completes the proof.

By the same method, we have the following Lemma 2.2 .

Lemma 2.2 Without loss of generality we assume that $k \leq 1$. Let $a_{1}=\min \left\{b_{1}, b_{2}\right\}, a_{2}=\max \left\{b_{1} e^{r_{1} \tilde{\tau}}, b_{2} e^{r_{2} \tilde{\tau}}\right\}$. For $\forall x_{0}>0, \quad y_{0}>0$, if $0<d_{1}+d_{2}<1$, then we have:

Case (1'). If $x_{0} \leq y_{0}, x_{0} \leq 1$, then $x_{n} \geq k x_{0}^{a_{2}^{n}}, y_{n} \geq x_{0}^{a_{2}^{n}}$, $n=1,2, \cdots$; if $x_{0} \leq y_{0}, x_{0}>1$, then $x_{n} \geq k x_{0}^{a_{1}^{n}}, y_{n} \geq x_{0}^{a_{1}^{n}}$, $n=1,2, \cdots$.
Case (2'). If $x_{0}>y_{0}, y_{0} \leq 1$, then $x_{n} \geq k y_{0}^{a_{2}^{n}}, \quad y_{n} \geq y_{0}^{a_{2}^{n}}$, $n=1,2, \cdots$; if $x_{0}>y_{0}, y_{0}>1$, then $x_{n} \geq k y_{0}^{a_{1}^{n}}, y_{n} \geq y_{0}^{a_{1}^{n}}$, $n=1,2, \cdots$.

Lastly, in order to establish the global stability of positively periodic solution, we introduce the following lemma:

Lemma 2.3 ([30]) Let $F: R_{+}^{n} \rightarrow R_{+}^{n}$ be continuous, $C^{1}$ in int $\left(R_{+}^{n}\right)$, and suppose $D F(0)$ exists with $\lim _{x \rightarrow 0^{+}} D F(z)=D F(0)$. In addition, assume

(a) $D F(x)>0$, if $x>0$;

(b) $D F(y)<D F(x)$, if $0<x<y$;

If $F(0)=0$, let $\lambda=\rho(D F(0))$. If $\lambda \leq 1$, then for every $x \geq 0, F^{n}(x) \rightarrow 0$ as $n \rightarrow \infty$; if $\lambda>1$, then either $F^{n}(x) \rightarrow \infty$ as $n \rightarrow \infty$ for every $x>0$; or there exists a unique nonzero fixed point $q$ of $F$. In the latter case, $q>0$ and for every $x>0, F^{n}(x) \rightarrow q$ as $n \rightarrow \infty$.

If $F(0) \neq 0$, then either $F^{n}(x) \rightarrow \infty$ as $n \rightarrow \infty$ for every $x \geq 0$ or there exists a unique fixed point $q$ of $F$. In the latter case, $q>0$ and for every $x>0$, $F^{n}(x) \rightarrow q$ as $n \rightarrow \infty$.

\section{Permanence}

The permanence plays an important role in mathematical ecology since the criterion of permanence for ecological systems is a condition ensuring the long-term survival of all species. In this section, we prove system (2.4) is permanent which will imply the permanence of system (1.4).

Theorem 3.1 For $\forall x_{0}>0, y_{0}>0$, system (2.4) is permanence if $0<d_{1}+d_{2}<1$.

Proof. Since $0<a_{1}, a_{2}<1, x_{0}>0, y_{0}>0$, we obtain

$$
\lim _{n \rightarrow \infty} x_{0}^{a_{1}^{n}}=1, \lim _{n \rightarrow \infty} x_{0}^{a_{2}^{n}}=1, \lim _{n \rightarrow \infty} y_{0}^{a_{1}^{n}}=1, \lim _{n \rightarrow \infty} y_{0}^{a_{2}^{n}}=1 .
$$

By Lemmas 2.1 and Lemmas 2.2, we can get that for $n \in N$, large enough, there exists constants $m_{i}$,

$M_{i}\left(0<m_{i}<M_{i}, i=1,2\right)$ such that $m_{1}<x_{n}<M_{1}$ and $m_{2}<y_{n}<M_{2}$, that is,

$m_{1} \leq \liminf _{t \rightarrow \infty} x_{n} \leq \limsup _{t \rightarrow \infty} x_{n} \leq M_{1}$ and

$m_{2} \leq \liminf _{t \rightarrow \infty} y_{n} \leq \limsup _{t \rightarrow \infty} y_{n} \leq M_{2}$ This means system (2.4) is permanent. The proof of Theorem 3.1 is completed.

\section{Existence and Uniqueness of Positive Periodic Solution}

In this part, we will prove the existence and uniqueness of the fixed points of system (2.4), which means that system (1.4) has a uniquely positive periodic solution.

Theorem 4.1 There exists a unique positive fixed point $\left(x^{*}, y^{*}\right)$ of system (2.4) provided that 


$$
\begin{aligned}
& 0<d_{1}+d_{2}<1,1-b_{1} \leq\left(1-b_{1} e^{r_{1} \tilde{\tau}}\right) e^{\left(r_{1}+r_{2}\right) \tilde{\tau}}, \\
& b_{1}+b_{2}+d_{1} \leq 1,1-b_{2} \leq\left(1-b_{2} e^{r_{2} \tilde{\tau}}\right) e^{\left(r_{1}+r_{2}\right) \tilde{\tau}} .
\end{aligned}
$$

Proof. Corresponding to (2.4), let us consider the following system (4.1):

$$
\left\{\begin{array}{l}
x=\left(1-d_{1}\right) x^{b_{1}}+d_{1} k y^{b_{2} e^{r_{2} \tilde{\tau}}}, \\
y=\left(1-d_{2}\right) y^{b_{2}}+\frac{d_{2}}{k} x^{b_{1} e^{r \tilde{\tau}}} .
\end{array}\right.
$$

From (4.1), we have

$$
\begin{aligned}
& x-\left(1-d_{1}\right) x^{b_{1}} \geq 0 \\
& y-\left(1-d_{2}\right) y^{b_{2}} \geq 0
\end{aligned}
$$

hence

$$
\left\{\begin{array}{l}
x \geq\left(1-d_{1}\right)^{\frac{1}{1-b_{1}}}=\xi \\
y \geq\left(1-d_{2}\right)^{\frac{1}{1-b_{2}}}=\eta .
\end{array}\right.
$$

From (4.1), we obtain

$$
\begin{aligned}
& \left\{\begin{array}{l}
y=\left[\frac{1}{k d_{1}}\left(x-\left(1-d_{1}\right) x^{b_{1}}\right)\right]^{\frac{1}{b_{2} e^{r_{2} \tilde{\tau}}}} \\
G(x, y)=\left(1-d_{2}\right) y^{b_{2}}-y+\frac{d_{2}}{k} x^{b_{1} e^{\eta \tilde{\tau}}} .
\end{array}\right. \\
& \frac{\mathrm{d} G}{\mathrm{~d} x}=\frac{1}{d_{1} k b_{2} e^{r_{2} \tilde{\tau}} y^{b_{2} e^{r_{2} \tilde{\tau}}-1}}\left\{\left[1-\left(1-d_{1}\right) b_{1} x^{b_{1}-1}\right]\left[\left(1-d_{2}\right) b_{2} y^{b_{2}-1}-1\right]+d_{1} d_{2} b_{1} b_{2} e^{\left(r_{1}+r_{2}\right) \tilde{\tau}} x^{b_{1} \eta^{\eta \tilde{\tau}}-1} y^{b_{2} e^{r_{2} \tilde{\tau}}-1}\right\}, \\
& \varphi(x, y)=\left[1-\left(1-d_{1}\right) b_{1} x^{b_{1}-1}\right]\left[\left(1-d_{2}\right) b_{2} y^{b_{2}-1}-1\right]+d_{1} d_{2} b_{1} b_{2} e^{\left(r_{1}+r_{2}\right) \tilde{\tau}} x^{b_{1} e^{r \tilde{\tau}}}-1 \\
& =-1+\left(1-d_{1}\right) b_{1} x^{b_{1}-1}+\left(1-d_{2}\right) b_{2} y^{b_{2}-1}-\left(1-d_{1}\right)\left(1-d_{2}\right) b_{1} b_{2} x^{b_{1}-1} y^{b_{2}-1}+d_{1} d_{2} b_{1} b_{2} e^{\left(r_{1}+r_{2}\right) \tilde{\tau}} x^{b_{1} \eta^{\eta \tilde{\tau}}-1} y^{b_{2} e^{r_{2} \tilde{\tau}}-1}, \\
& \frac{\mathrm{d} \varphi(x)}{\mathrm{d} x}=\left(1-d_{1}\right) b_{1}\left(b_{1}-1\right) x^{b_{1}-2}+\left(1-d_{2}\right) b_{2}\left(b_{2}-1\right) y^{b_{2}-2} \frac{\mathrm{d} y}{\mathrm{~d} x} \\
& -\left(1-d_{1}\right)\left(1-d_{2}\right) b_{1} b_{2}\left[\left[b_{1}-1\right] x^{b_{1}-2} y^{b_{2}-1}+\left(b_{2}-1\right) x^{b_{1}-1} y^{b_{2}-2} \frac{\mathrm{d} y}{\mathrm{~d} x}\right] \\
& +d_{1} d_{2} b_{1} b_{2} e^{\left(r_{1}+r_{2}\right) \tilde{\tau}}\left[\left(b_{1} e^{r_{1} \tilde{\tau}}-1\right) x^{b_{1} e^{r^{r} \tilde{\tau}}-2} y^{b_{2} e^{r_{2} \tilde{\tau}}-1}+\left(b_{2} e^{r_{2} \tilde{\tau}}-1\right) x^{b_{1} e^{\eta \tilde{\tau}}-1} y^{b_{2} e^{r_{2} \tilde{\tau}}-2} \frac{\mathrm{d} y}{\mathrm{~d} x}\right] \\
& =b_{1}\left(b_{1}-1\right) x^{b_{1}-2} \frac{1}{1-d_{2}}\left[\left(1-d_{1}\right)\left(1-d_{2}\right)-b_{2}\left(1-d_{1}-d_{2}\right)\left(1-d_{2}\right) y^{b_{2}-1}\right] \\
& +b_{2}\left(b_{2}-1\right) y^{b_{2}-2} \frac{\mathrm{d} y}{\mathrm{~d} x} \frac{1}{1-d_{1}}\left[\left(1-d_{1}\right)\left(1-d_{2}\right)-b_{1}\left(1-d_{1}-d_{2}\right)\left(1-d_{1}\right) x^{b_{1}-1}\right] \\
& +d_{1} d_{2} b_{1} b_{2}\left[e^{\left(r_{1}+r_{2}\right) \tilde{\tau}}\left(b_{1} e^{r_{1} \tilde{\tau}}-1\right) x^{b_{1} e^{n \tilde{\tau}}-2} y^{b_{2} e^{r^{2} \tilde{\tau}}-1}-\left(b_{1}-1\right) x^{b_{1}-2} y^{b_{2}-1}\right] \\
& +d_{1} d_{2} b_{1} b_{2} \frac{\mathrm{d} y}{\mathrm{~d} x}\left[e^{\left(r_{1}+r_{2}\right) \tilde{\tau}}\left(b_{2} e^{r_{2} \tilde{\tau}}-1\right) x^{b_{1} e^{\eta_{1} \tilde{\tau}}}-1 y^{b_{2} e^{r_{2} \tilde{\tau}}-2}-\left(b_{2}-1\right) x^{b_{1}-1} y^{b_{2}-2}\right] . \\
& \left\{\begin{array}{l}
\frac{\mathrm{d} y}{\mathrm{~d} x}=\frac{1-\left(1-d_{1}\right) b_{1} x^{b_{1}-1}}{d_{1} k b_{2} e^{r_{2} \tilde{\tau}} y^{b_{2} e^{r_{2}} \tilde{\tau}}-1}, \\
\frac{\mathrm{d} G}{\mathrm{~d} x}=\left[\left(1-d_{2}\right) b_{2} y^{b_{2}-1}\right] \frac{\mathrm{d} y}{\mathrm{~d} x}-\frac{\mathrm{d} y}{\mathrm{~d} x}+\frac{d_{2} b_{1} e^{r_{1} \tilde{\tau}}}{k} x^{b_{1} e^{r^{n} \tilde{\tau}}-1},
\end{array}\right.
\end{aligned}
$$

It follows from (4.3) that we obtain 
By (4.2) and assumption, we have $\left(1-d_{1}\right) x^{b_{1}-1} \leq 1$, $\left(1-d_{2}\right) y^{b_{2}-1} \leq 1, \quad 1-b_{1} \leq\left(1-b_{1} e^{r_{1} \tilde{\tau}}\right) e^{\left(\eta_{1}+r_{2}\right) \tilde{\tau}}$,

$1-b_{2} \leq\left(1-b_{2} e^{r_{2} \tilde{\tau}}\right) e^{\left(r_{1}+r_{2}\right) \tilde{\tau}}$. So, we obtain $\frac{\mathrm{d} \varphi(x)}{\mathrm{d} x}<0$, which implies that $\varphi(x)$ is a decreasing function on the interval $[\xi,+\infty)$.

Since

$$
\begin{aligned}
& \varphi(1)=-1+\left(1-d_{1}\right) b_{1} \\
& +\left(1-d_{2}\right) b_{2} k^{\frac{1-b_{2}}{b_{2} e^{2 \tau}}}\left[1-\left(1-d_{1}\right) b_{1}\right] \\
& +d_{1} d_{2} b_{1} b_{2} e^{\left(r_{1}+r_{2}\right) \tilde{\tau}} k^{\frac{1-b_{2} r^{r_{2} \tilde{\tau}}}{b_{2} e^{r_{2} \tilde{\tau}}}} \\
& <-1+b_{1}+b_{2}+d_{1} \leq 0, \\
& y(\xi)=0, \\
& \lim _{x \rightarrow \xi^{+}} \varphi(x)=\lim _{x \rightarrow \xi^{+}}\left\{-1+\left(1-d_{1}\right) b_{1} x^{b_{1}-1}\right. \\
& +\left(1-d_{2}\right) b_{2} y^{b_{2}-1}\left[1-\left(1-d_{1}\right) b_{1} x^{b_{1}-1}\right] \\
& \left.+d_{1} d_{2} b_{1} b_{2} e^{\left(r_{1}+r_{2}\right) \tilde{\tau}} x^{b_{1} e^{\eta^{n} \tilde{\tau}}-1} y^{b_{2} e^{r_{2} \tilde{\tau}}-1}\right\} \\
& =+\infty \text {, }
\end{aligned}
$$

using the intermediate value theorem, there exists a unique point $\xi_{1} \in(\xi, 1)$ such that $\varphi\left(\xi_{1}\right)=0$. Besides,

thus

$$
\begin{aligned}
& \varphi(x)>0, \forall x \in\left(\xi, \xi_{1}\right), \\
& \varphi(x)<0, \forall x \in\left(\xi_{1},+\infty\right),
\end{aligned}
$$

$$
\begin{aligned}
& \frac{\mathrm{d} G(x)}{\mathrm{d} x}>0, \forall x \in\left(\xi, \xi_{1}\right), \\
& \frac{\mathrm{d} G(x)}{\mathrm{d} x}<0, \forall x \in\left(\xi_{1},+\infty\right),
\end{aligned}
$$

which, together with $G(\xi)>0$, leads to $G(x)>0$, $\forall x \in\left(\xi, \xi_{1}\right)$. By $G\left(\xi_{1}\right)>0, G(1)<0$, we have that there exists a unique point $x^{*} \in(\xi, 1)$ such that $G\left(x^{*}, y^{*}\right)=0$. The proof is completed.

\section{Global Stability}

Now, we will prove that the positive fixed point $\left(x^{*}, y^{*}\right)$ of system (4.1) is globally stable by using Lemma 2.3, which means that the positive periodic solution of system (1.4) is globally stable.

Theorem 5.1 For every $(x, y)>(0,0)$, $F^{n}(x) \rightarrow\left(x^{*}, y^{*}\right)$ as $n \rightarrow \infty$.

Proof. For any small $\varepsilon_{1}>0, \varepsilon_{2}>0$, we make the change of variable

$$
x=u+\varepsilon_{1}, y=v+\varepsilon_{2},
$$

and get the map $F(u, v)=\left(f_{1}(u, v), f_{2}(u, v)\right)$, that is,

$$
\left\{\begin{array}{l}
u=\left(1-d_{1}\right)\left(u+\varepsilon_{1}\right)^{b_{1}}+d_{1} k\left(v+\varepsilon_{2}\right)^{b_{2} e^{r_{2} \tilde{z}}}-\varepsilon_{1}=f_{1}(u, v), \\
v=\left(1-d_{2}\right)\left(v+\varepsilon_{2}\right)^{b_{2}}+\frac{d_{2}}{k}\left(u+\varepsilon_{1}\right)^{b_{1} e^{\eta^{\tilde{\tau}}}}-\varepsilon_{2}=f_{2}(u, v) .
\end{array}\right.
$$

Now, we show that $F(u, v)$ satisfies the hypotheses of Lemma 2.3. It is easy to see that $F(u, v)$ is continuous $C^{1}$ in $\operatorname{int}\left(R_{+}^{2}\right)$, and $F(0,0) \neq 0$.

Since

$$
\begin{aligned}
& \text { DF }(u, v) \\
& =\left(\begin{array}{ll}
\left(1-d_{1}\right) b_{1}\left(u+\varepsilon_{1}\right)^{b_{1}-1} & d_{1} b_{2} k e^{r_{2} \tilde{\tau}}\left(v+\varepsilon_{2}\right)^{b_{2} e^{r_{2} \tilde{\tau}}-1} \\
\frac{d_{2} b_{1} e^{\eta \tilde{\tau}}}{k}\left(u+\varepsilon_{1}\right)^{b_{1} e^{\eta \tilde{\tau}}-1} & \left(1-d_{2}\right) b_{2}\left(v+\varepsilon_{2}\right)^{b_{2}-1}
\end{array}\right), \\
& D F(0,0)=\left(\begin{array}{ll}
\left(1-d_{1}\right) b_{1} \varepsilon_{1}^{b_{1}-1} & d_{1} b_{2} k e^{r_{2} \tilde{\tau}} \varepsilon_{2}^{b_{2} e^{r_{2} \tilde{\tau}}-1} \\
\frac{d_{2} b_{1} e^{r_{1} \tilde{\tau}}}{k} \varepsilon_{1}^{b_{1} e^{\eta \tilde{\tau}}-1} & \left(1-d_{2}\right) b_{2} \varepsilon_{2}^{b_{2}-1}
\end{array}\right),
\end{aligned}
$$

so $\lim _{(u, v) \rightarrow\left(0^{+}, 0^{+}\right)} D F(u, v)=D F(0,0)$. If $(u, v)>0$, then $D F(u, v)>0$; if $(0,0)<\left(u_{1}, v_{1}\right)<\left(u_{2}, v_{2}\right)$, then $D F\left(u_{2}, v_{2}\right)<D F\left(u_{1}, v_{1}\right)$. Obviously, Theorem 5.1 satisfies all the conditions of Lemma 2.3 , then for every $u>0, v>0$, we have $F^{n}(u, v) \rightarrow\left(x^{*}-\varepsilon_{1}, y^{*}-\varepsilon_{2}\right)$ as $n \rightarrow \infty$. Corresponding to $x-y$ coordinate, this means for $x>\varepsilon_{1}, y>\varepsilon_{2}$, the system (4.1) trends to unique fixed point.

Besides, we have $x_{n}>\varepsilon_{1}, y_{n}>\varepsilon_{2}$ for initial value $\left(x_{0}, y_{0}\right)>(0,0)$ as $n \rightarrow \infty$.

From the above analysis, we can know that for every $\left(x_{0}, y_{0}\right)>0$, the trajectory of system (2.4) will trend to $\left(x^{*}, y^{*}\right)$. This completes the proof.

\section{Numerical Simulation and Discussion}

In this paper, we have investigated a single species model with symmetric bidirectional impulsive diffusion and dispersal delay, the criteria for the permanence, existence, uniqueness and stability of positive periodic solution are established.

Firstly, in Table 1 we show the effect of time delay on the populations. By simple calculating, we can get

$$
\begin{aligned}
& 1-b_{1} \leq\left(1-b_{1} e^{r_{1} \tilde{\tau}}\right) e^{\left(r_{1}+r_{2}\right) \tilde{\tau}}, \\
& 1-b_{2} \leq\left(1-b_{2} e^{r_{2} \tilde{\tau}}\right) e^{\left(r_{1}+r_{2}\right) \tilde{\tau}},
\end{aligned}
$$

provided $0<\tilde{\tau}<10$. And

$$
d_{1}+d_{2}=0.9<1, b_{1}+b_{2}+d_{1}=0.3001<1 .
$$

So, all conditions of Theorem 3.1 and Theorem 4.1 hold, which means system (2.2) is permanent and has an unique globally stable periodic solution. From Figure 1, we can see that in this four different cases the species $x$ 


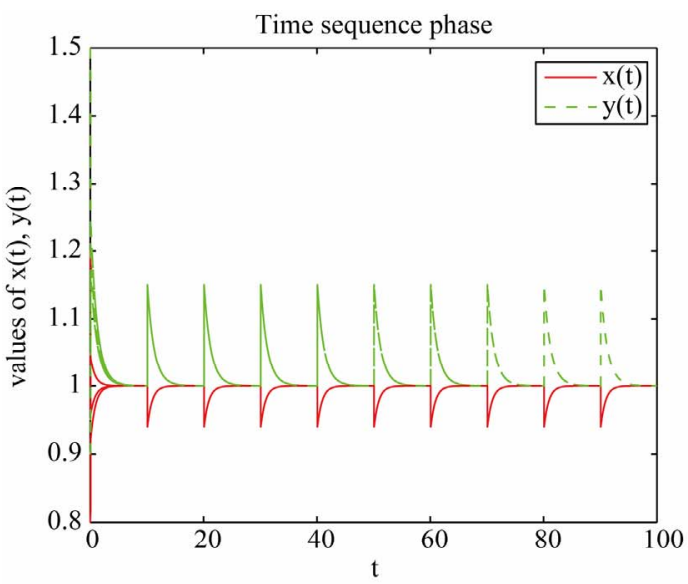

(a)

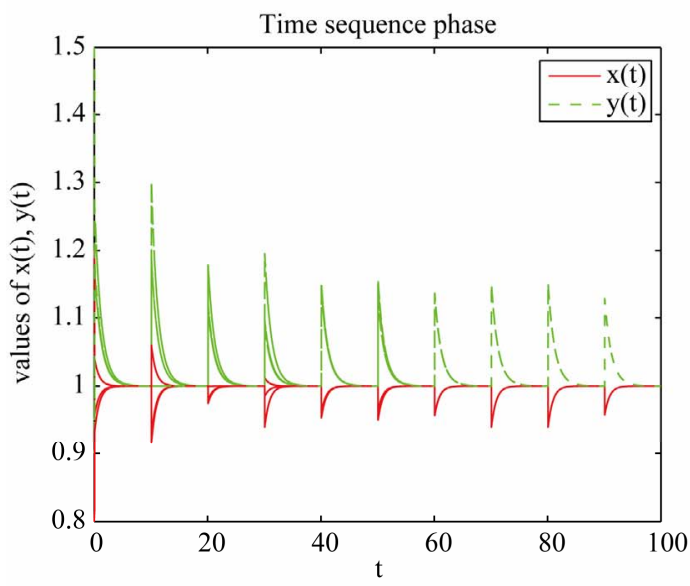

(c)

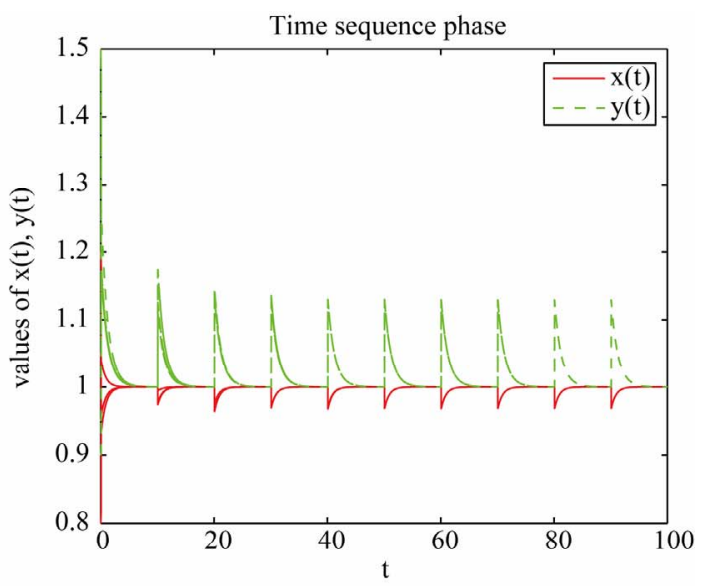

(b)

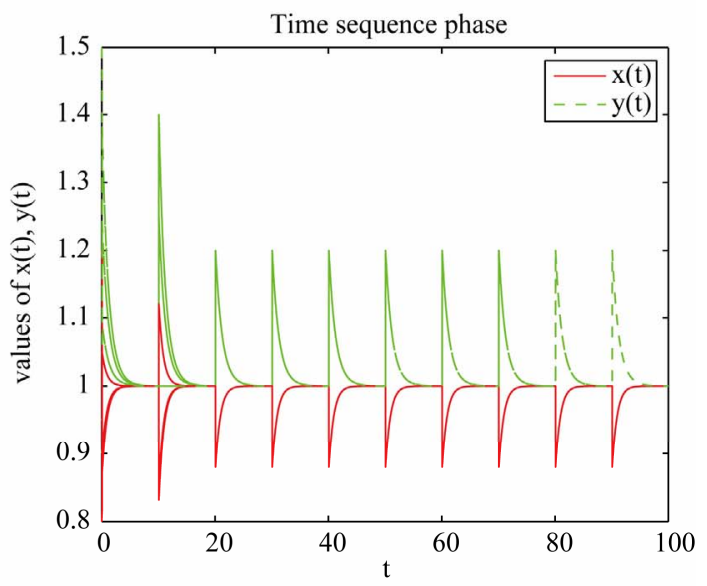

(d)

Figure 1. Dynamical behavior of system (2.2). Here, we take three sets of initial values $(0.8,1.5),(1.2,0.9),(1.05,0.95)$.

Table 1. Parameter values used in the simulations of model (2.2).

\begin{tabular}{|c|c|c|}
\hline Parameter & Interpretation & Value \\
\hline$k_{1}$ & Carrying capacity of populations in Patch 1 & 1000 \\
\hline$k_{2}$ & Carrying capacity of populations in Patch 2 & 800 \\
\hline$r_{1}$ & Intrinsic growth rate of populations in Patch 1 & 1.2 \\
\hline$r_{2}$ & Intrinsic growth rate of populations in Patch 2 & 0.8 \\
\hline$d_{1}$ & $\begin{array}{l}\text { Emigrant rate of populations from } \\
\text { Patch } 1 \text { to Patch } 2\end{array}$ & 0.3 \\
\hline$d_{2}$ & $\begin{array}{l}\text { Emigrant rate of populations from } \\
\text { Patch } 2 \text { to Patch } 1\end{array}$ & 0.6 \\
\hline$\tau$ & $\begin{array}{c}\text { The period of dispersal between two pulse } \\
\text { events }\end{array}$ & 10 \\
\hline
\end{tabular}

and $y$ are both permanent. Moreover, the longer the duration of the time delay $(0<\tilde{\tau}<\tau)$, the larger the limit inferior of $x$ and the lower the limit superior of $y$ (see Figures 1(a) and (b)). This implies the case with dispersal delay is beneficial to species $x$ living and harmful to species $y$.

Next, in Table 2, if $\tilde{\tau}>\tau$, species $x$ and $y$ are both permanent, too (see Figure 1(c)). If we take $d_{1}=0.6$, $d_{2}=0.8, \tilde{\tau}=12$, and keep other parameters unchanged in Table 1, then $d_{1}+d_{2}=1.4>1$ which purports that it does not satisfy the condition of Theorem 3.1, but we also get species $x$ and $y$ are both permanent (see Figure 1(d)). Therefore, $0<\tilde{\tau}<\tau$ and $0<d_{1}+d_{2}<1$ are sufficient conditions for the permanence of system (1.4), but not a necessary ones.

Furthermore, in Table 3, by numerical simulations (see Figures 2(a) and (b)), we find that all of the solutions of system (2.2) which through these initial points will converge to the positive periodic solution. Therefore, we can conclude that under the assumptions of Theorem (4.1) system (2.2) has a unique positive periodic solution which is globally asymptotically stable. In addition, the periodic solution is lager in Case II than in Case I which indicates that the duration of the time delay is beneficial to species $x$ living again. Comparing Figures 2(c) and (d), we realize that system (1.4) with time delay is more complicated than without. 


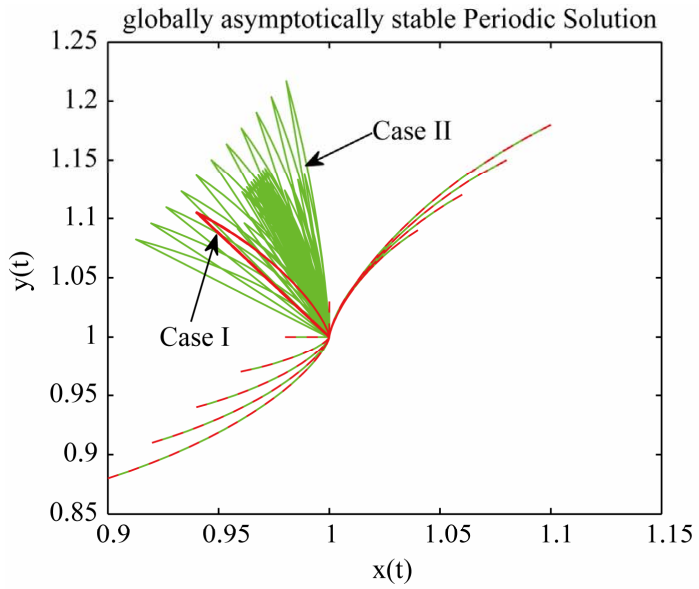

(a)

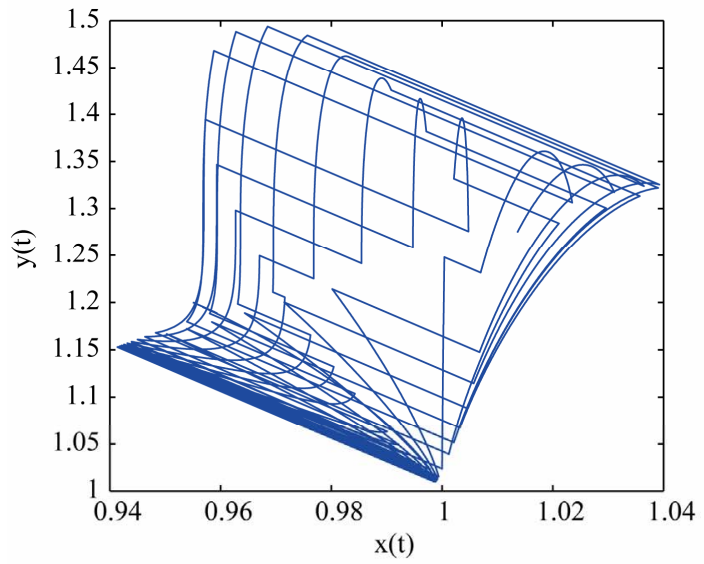

(c)

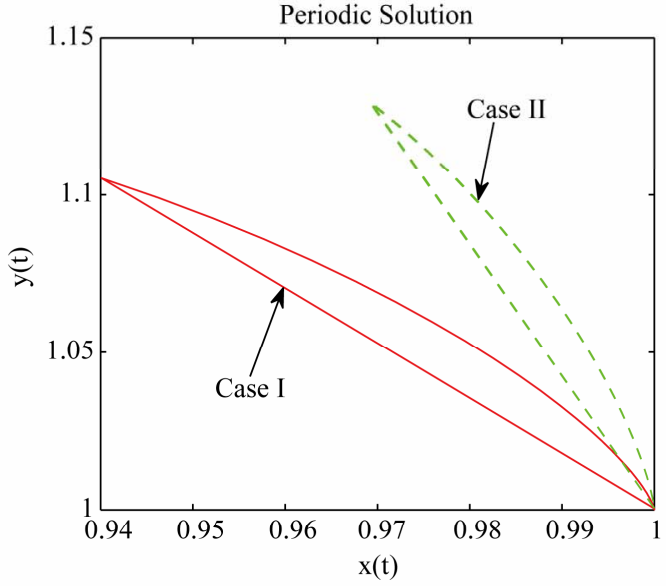

(b)

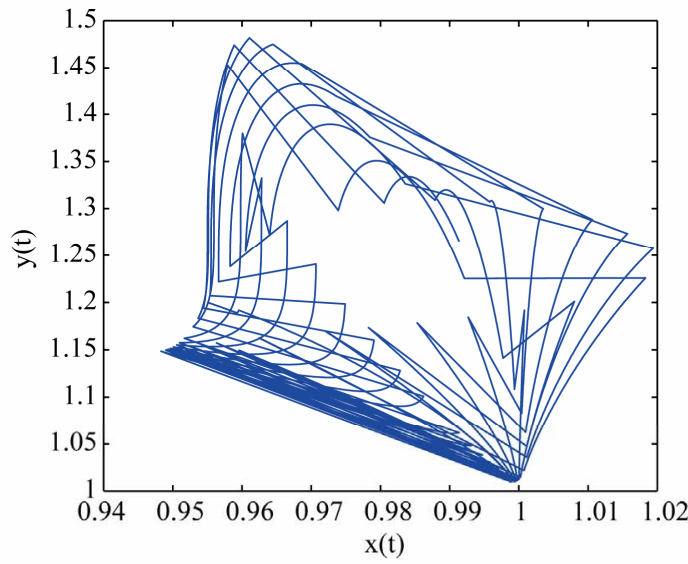

(d)

Figure 2. Dynamical behavior of system (2.2). we take a series initial points, such as $(0.9,0.88),(0.92,0.9),(1,1.03)$.

Table 2. Simulations of model (2.2).

\begin{tabular}{ccccc}
\hline Case & $\tilde{\tau}$ & $x_{1}$ & $x_{2}$ & Figure \\
\hline 1 & 0 & Permanent & Permanent & Figure 1(a) \\
2 & 9.9 & Permanent & Permanent & Figure 1(b) \\
3 & 20 & Permanent & Permanent & Figure 1(c) \\
4 & 12 & Permanent & Permanent & Figure 1(d) \\
\hline
\end{tabular}

Table 3. Simulations of model (2.2).

\begin{tabular}{cccccc}
\hline Case & $\tau$ & $\tilde{\tau}$ & $r_{1}$ & $r_{2}$ & Figure \\
\hline I & 10 & 0 & $1+\sin \left(\frac{1}{5} \pi t\right)$ & $\frac{1}{2}+\sin \left(\frac{1}{5} \pi t\right)$ & $\begin{array}{c}\text { Figures 2(a) and } \\
\text { (b) }\end{array}$ \\
II & 10 & 9.9 & $1+\sin \left(\frac{1}{5} \pi t\right)$ & $\frac{1}{2}+\sin \left(\frac{1}{5} \pi t\right)$ & $\begin{array}{c}\text { Figures 2(a) and } \\
\text { (b) }\end{array}$ \\
III & 1.915 & 0 & $1+\sin \left(\frac{1}{5} \pi t\right)$ & $\frac{1}{2}+\sin \left(\frac{1}{5} \pi t\right)$ & Figure 2(c) \\
IV & 1.915 & 1.03 & $1+\sin \left(\frac{1}{5} \pi t\right)$ & $\frac{1}{2}+\sin \left(\frac{1}{5} \pi t\right)$ & Figure 2(d) \\
\hline
\end{tabular}




\section{Acknowledgements}

This work was supported by the National Natural Science Foundation of P.R. China (10901130, 10961022), Natural Science Foundation of Xinjiang Province of China (2012211B07), the China Scholarship Council.

\section{REFERENCES}

[1] Z. Teng and Z. Lu, "The Effect of Dispersal on SingleSpecies Nonautonomous Dispersal Models with Delays," Journal of Mathematical Biology, Vol. 42, No. 5, 2001, pp. 439-454. doi:10.1007/s002850000076

[2] Z. Teng and L. Chen, "Permanence and Extinction of Periodic Predator-Prey Systems in a Patchy Environment with Delay," Nonlinear Analysis: Real World Applications, Vol. 4, No. 2, 2003, pp. 335-364. doi:10.1016/S1468-1218(02)00026-3

[3] L. Buttel, R. Durrett and S. Levin, "Competition and Species Packing in Patchy Environments," Theoretical Population Biology, Vol. 61, No. 3, 2002, pp. 265-276. doi:10.1006/tpbi.2001.1569

[4] J. Cui, Y. Takeuchi and Z. Lin, "Permanence and Extinction for Dispersal Population Systems," Journal of Mathematical Analysis and Applications, Vol. 298, No. 1, 2004, pp. 73-93. doi:10.1016/j.jmaa.2004.02.059

[5] Y. Takeuchi, "Diffusion Effect on Stability of LotkaVolterra Models," Bulletin of Mathematical Biology, Vol. 48, No. 5-6, 1986, pp. 585-601.

[6] Y. Takeuchi, J. Cui, R. Miyazak and Y. Saito, "Permanence of Delayed Population Model with Dispersal Loss," Mathematical Biosciences, Vol. 201, No. 1-2, 2006, pp. 143-156. doi:10.1016/j.mbs.2005.12.012

[7] E. Beretta and Y. Takeuchi, "Global Stability of SingleSpecies Diffusion Volterra Models with Continuous Time Delays," Bulletin of Mathematical Biology, Vol. 49, 1987, pp. 431-448.

[8] E. Beretta and Y. Takeuchi, "Global Asymptotic Stability of Lotka-Volterra Diffusion Models with Continuous Time Delays," SIAM Journal on Applied Mathematics, Vol. 48, No. 3, 1998, pp. 627-651. doi:10.1137/0148035

[9] E. Beterra, P. Fergola and C. Tenneriello, "Ultimate Boundedness of Nonautonomous Diffusive Lotka-Volterra Patches," Mathematical Biosciences, Vol. 92, No. 1, 1988, pp. 29-53. doi:10.1016/0025-5564(88)90004-1

[10] H. I. Freedman, J. Shukla and Y. Takeuchi, "Population Diffusion in a Two-Patch Environment," Mathematical Biosciences, Vol. 95, No. 1, 1989, pp. 111-123. doi:10.1016/0025-5564(89)90055-2

[11] A. Hastings, "Dynamics of a Single Species in a Spatially Varying Environment: The Stability Role of High Dispersal Rates," Journal of Mathematical Biology, Vol. 16, No. 1, 1982, pp. 49-55. doi:10.1007/BF00275160

[12] W. Wang and L. Chen, "Global Stability of a Population Dispersal in a Two-Patch Environment," Dynamic Systems \& Applications, Vol. 6, 1997, pp. 207-216.

[13] L. Zhang and Z. Teng, "Permanence for a Class of Peri- odic Time-Dependent Competitive System with Delays and Dispersal in a Patchy-Environment," Applied Mathematics and Computation, Vol. 188, No. 1, 2007, pp. 855864. doi:10.1016/j.amc.2006.10.037

[14] L. Zhang and Z. Teng, "Permanence for a Delayed Periodic Predator-Prey Model with Prey Dispersal in MultiPatches and Predator Density-Independent," Journal of Mathematical Analysis and Applications, Vol. 338, No. 1, 2008, pp. 175-193. doi:10.1016/j.jmaa.2007.05.016

[15] E. Beretta, F. Solimano and Y. Takeuchi, "Global Stability and Periodic Orbits for Two Patch Predator-Prey Diffusion-Delay Models," Mathematical Biosciences, Vol. 85 , No. 2, 1987, pp. 153-183. doi:10.1016/0025-5564(87)90051-4

[16] R. Mahbuba and L. Chen, "On the Nonautonomous Lotka-Volterra Competion System with Diffusion," Differential Equations and Dynamical Systems, Vol. 2, 1994, pp. 243-253.

[17] J. G. Skellam, "Random Dispersal in Theoretical Population," Biometrika, Vol. 38, 1951, pp. 196-218.

[18] J. Hui and L. Chen, "A Single Species Model with Impulsive Diffusion," Acta Mathematicae Applicatae Sinica. English Series, Vol. 21, No. 1, 2005, pp. 43-48. doi:10.1007/s10255-005-0213-3

[19] L. Wang and L. Chen, "Impulsive Diffusion in Single Species Model," Chaos Solitons, Fractals, Vol. 33, No. 4, 2007, pp. 1213-1219. doi:10.1016/j.chaos.2006.01.102

[20] A. Lakmeche and O. Arino, "Bifurcation of Nontrivial Periodic Solution of Impulsive Differential Equations Arising Chemotherapeutic Treatment," Dynamics of Continuous, Discrete and Impulsive Systems, Vol. 7, 2000, pp. 265-287.

[21] L. Zhang and Z. Teng, "N-Species Non-Autonomous Lotka-Volterra Competitive Systems with Delays and Impulsive Perturbations," Nonlinear Analysis: Real World Applications, Vol. 12, No. 6, 2011, pp. 3152-3169. doi:10.1016/j.nonrwa.2011.05.015

[22] J. Vandermeer, L. Stone and B. Blasius, "Categories of Chaos and Fractal Basin Boundaries in Forced Predator-Preymodels," Chaos Solitons, Fractals, Vol. 12, No. 2, 2001, pp. 265-276. doi:10.1016/S0960-0779(00)00111-9

[23] L. Dong, L. Chen and L. Sun, "Optimal Harvesting Policy for Inshore-Offshore Fishery Model with Impulsive Diffusion," Acta Mathematica Scientia, Vol. 27, No. 2, 2007, pp. 405-412. doi:10.1016/S0252-9602(07)60040-X

[24] Z. Zhao, X. Zhang and L. Chen, "The Effect of Pulsed Harvesting Policy on the Inshore-Offshore Fishery Model with the Impusive Diffusion," Nonlinear Dynamic, Vol. 63, No. 4, 2011, pp. 537-545. doi:10.1007/s11071-009-9527-7

[25] Y. Kuang, "Delay Differential Equations with Applications in Population Dynamics," Academic Press, New York, 1993.

[26] X. Zhao, "Dynamical Systems in Population Biology," Springer-Verlag, New York, 2003.

[27] H. L. Smith, "Monotone Dynamical Systems: An Introduction to the Theory of Competitive and Cooperative 
Systems," Mathematical Surveys and Monographs, Vol. 41, 1995.

[28] X. Zhao, "Global Attractivity in a Class of Non-Monotone Reaction? Diffusion Equations with Time Delay," Canadian Applied Mathematics Quarterly, Vol. 17, 2009, pp. 271-281.

[29] X. Meng and L. Chen, "Permanence and Global Stability in an Impulsive Lotka-Volterra N-Species Competitive System with Both Discrete Delays and Continuous Delays," International Journal of Biomathematics, Vol. 1, No. 2, 2008, pp. 179-196.

\section{doi:10.1142/S1793524508000151}

[30] H. L. Smith, "Cooperative Systems of Differential Equations with Concave Nonlinearities," Nonlinear Analysis: Theory, Methods \& Applications, Vol. 10, 1986, pp. 1037-1052.

[31] V. Lakshmikantham, D. D. Bainov and P. S. Simeonov, "Theory of Impulsive Differential Equations," World Scientific, Singapore, 1989.

[32] D. Bainov and P. Simeonov, "Impulsive Differential Equations: Periodic Solutions and Applications," Longman, England, 2003. 